\title{
MORE ON BASES OF UNCOUNTABLE FREE ABELIAN GROUPS
}

\author{
NOAM GREENBERG, LINUS RICHTER, SAHARON SHELAH, AND DAN TURETSKY
}

\begin{abstract}
We extend results found by Greenberg, Turetsky, and Westrick in [7] and investigate effective properties of bases of uncountable free abelian groups. Assuming $V=L$, we show that if $\kappa$ is a regular uncountable cardinal and $X$ is a $\Delta_{1}^{1}\left(L_{\kappa}\right)$ subset of $\kappa$, then there is a $\kappa$-computable free abelian group whose bases cannot be effectively computed by $X$. Unlike in [7], we give a direct construction.
\end{abstract}

\section{INTRODUCTION}

The recursive construction of a basis of a vector space is taught to first-year students of linear algebra: at each step, add some vector which does not lie in the span of the previous ones chosen. This "algorithm" works equally well in finite, countable and uncountable-dimensional vector spaces. In the uncountable case, this can be made precise using the framework introduced by Greenberg and Knight for uncountable computable structure theory, using admissible computability on uncountable cardinals [6]. In fact, just like the countable case, a single Turing jump (relative to the diagram of the vector space) suffices. In particular, for every cardinal $\kappa$, every $\kappa$-computable vector space (over a $\kappa$-computable field) has a basis which is definable in $L_{\kappa}$.

What happens when we consider free abelian groups, objects which appear just as simple as vector spaces? In the countable case, based on a strong notion of independence introduced by Pontryagin [10], a similar algorithm can be performed; Downey and Melnikov [1] used this notion to show that a single Turing jump suffices to build a basis of a countable free abelian group. This algorithm, however, fails badly in the uncountable case, because difficulties can arise in limit steps: it is possible to generate an $\omega$-sequence $b_{0}, b_{1}, \ldots$ of elements of a free abelian group such that every finite initial segment of the sequence is extendible to a basis of the group, but the whole sequence cannot. That is, the construction, which appears perfectly fine at every finite step, "explodes" at the limit step.

In [7], the authors showed that this difficulty is fundamental: in general, there is no way to construct bases of free abelian groups in a step-by-step recursive, or definable, construction. Formally, what they showed (under the standard assuption that $V=L$ ) is that for most uncountable regular cardinals $\kappa$, there are $\kappa$-computable free abelian groups which have no bases definable over $L_{\kappa}$; in fact,

2010 Mathematics Subject Classification. Primary: 03C57. Secondary: 03D60.

Key words and phrases. Free abelian groups,admissible computability, uncountable computable structure theory.

Greenberg was partially supported by a Marsden grant from the Royal Society of New Zealand, and by a Lady Davis Visiting Professorship. Shelah was partially supported by the European Research Council grant 338821. Paper 1188 on Shelah's list. 
no single $\kappa$-Turing degree which is $\Delta_{1}^{1}\left(L_{\kappa}\right)$ can serve as an oracle which computes bases for all $\kappa$-computable free abelian groups. This result has two shortcomings:

(1) It does not apply to all regular uncountable cardinals $\kappa$;

(2) It does not rely on a direct construction.

These two are related. The argument in [7] relies on the complexity of identifying which groups are free. The authors show that if $\kappa$ is not weakly compact, then the collection of free abelian groups is $\boldsymbol{\Sigma}_{1}^{1}\left(L_{\kappa}\right)$-complete, and in many cases (such as successor cardinals, or the least inaccessible), is (lighface) $\Sigma_{1}^{1}\left(L_{\kappa}\right)$-complete. In these cases, this completeness result implies that searching for bases cannot be restricted to the sets computable from a fixed $\Delta_{1}^{1}\left(L_{\kappa}\right)$ oracle. It is not clear from the construction, though, how to directly build, given a $\Delta_{1}^{1}\left(L_{\kappa}\right)$ oracle $X$, a $\kappa$ computable free abelian group $G$ with no $X$-computable basis. And this approach cannot work for weakly compact cardinals $\kappa$; the very compactness property of these cardinals implies that it is in fact not very difficult to identify which groups are free; in fact, a single jump suffices. This leaves the original question, of the complexity of bases, open for these cardinals.

In the current paper we fully answer the original question:

Theorem $1.1(V=L)$. Let $\kappa$ be regular and uncountable. For any $X \in 2^{\kappa}$ which is $\Delta_{1}^{1}\left(L_{\kappa}\right)$ there is a $\kappa$-computable free abelian group, none of whose bases is $X$-computable.

The proof of the theorem is done by a direct construction.

\section{Preliminaries, AND the Plan of the Proof}

The proof of Theorem 1.1 is the combination of two distinct parts: we will first reduce the problem to one in "computable set theory", one which abstracts away the algebraic part; and then give a construction solving the reduced problem. Throughout the paper, we assume $V=L$. We refer the reader to [6] for the basic definitions of $\kappa$-computability. A general reference for torsion-free abelian groups is $[5]$

We start by recalling some basic concepts that were used in [7].

All groups we will discuss are abelian and torsion-free. A group $G$ is free abelian if it has a basis: a subset $B$ which is linearly independent $\left(\sum m_{i} b_{i}=0\right.$ implies each $m_{i}=0$, where $m_{i} \in \mathbb{Z}$ and $b_{i} \in B$ ) and spans $G$ (every element of $G$ is of the form $\sum m_{i} b_{i}$ for some $m_{i} \in \mathbb{Z}$ and $b_{i} \in B$ ). We will omit the adjective "abelian" and just call these groups free.

Definition 2.1. Let $G$ be a group. A subgroup $H$ of $G$ detaches in $G$ if there is a $G$-subgroup $K$ such that $G=H \oplus K$. We write $H \mid G$.

Any subgroup of a free abelian group is free. A subgroup $H$ of a free abelian group $G$ detaches in $G$ if and only if some (equivalently any) basis of $H$ can be extended to a basis of $G$, if and only if the quotient $G / H$ is free. If $H$ detaches in $G$ then $H$ detaches in any subgroup $K$ of $G$ containing $H$.

Definition 2.2. A sequence $\left\langle G_{\alpha}\right\rangle_{\alpha<\gamma}$ of groups of some ordinal length $\gamma$ is increasing if $\alpha<\beta$ implies $G_{\alpha} \subseteq G_{\beta}$; it is continuous if for all limit $\alpha<\gamma, G_{\alpha}=\bigcup_{\beta<\alpha} G_{\beta}$. A filtration of a group $G$ is a sequence $\bar{G}=\left\langle G_{\alpha}\right\rangle$ such that $\bar{G}^{\wedge}\langle G\rangle$ is increasing and continuous, and $\left|G_{\alpha}\right| \leqslant|\alpha|$ for all $\alpha$. 
If $\gamma$ is regular and $G$ is a group of universe $\gamma$ then all filtrations of $G$ agree on a club; in fact, for club many $\alpha, G_{\alpha}=G \cap \alpha$.

Definition 2.3. Let $\bar{G}=\left\langle G_{\alpha}\right\rangle_{\alpha<\gamma}$ be increasing and continuous. The detachment set of $\bar{G}$ is

$$
\operatorname{Div}(\bar{G})=\left\{\alpha<\gamma: \forall \beta \in(\alpha, \gamma)\left(G_{\alpha} \mid G_{\beta}\right)\right\}
$$

If $\gamma$ is regular and $\bar{G}, \bar{G}^{\prime}$ are two filtrations of a group of universe $\gamma$, then $\operatorname{Div}(\bar{G})$ and $\operatorname{Div}\left(\bar{G}^{\prime}\right)$ agree on a club.

The following can essentially be found in [2]; see [3, IV.1.7].

Proposition 2.4. Suppose $\gamma$ is a limit ordinal and let $\bar{G}=\left\langle G_{\alpha}\right\rangle_{\alpha<\gamma}$ be a filtration of a group $G_{\gamma}$. Suppose $G_{\alpha}$ is free for all $\alpha<\gamma$.

(1) If $\operatorname{Div}(\bar{G})$ contains a $\gamma$-club, then $G_{\gamma}$ is free.

(2) If $\gamma$ is regular and $G_{\gamma}$ is free, then $\operatorname{Div}(\bar{G})$ contains a $\gamma$-club.

Remark 2.5. Let $\gamma$ be a limit ordinal; let $\bar{G}=\left\langle G_{\alpha}\right\rangle_{\alpha<\gamma}$ be a filtration of a group $G_{\gamma}$. Suppose that $\operatorname{Div}(\bar{G})$ contains a club of $\gamma$. Then

$$
\operatorname{Div}(\bar{G})=\left\{\alpha<\gamma: G_{\alpha} \mid G_{\gamma}\right\}
$$

The proof of Proposition 2.4 is effective. This gives the following:

Proposition 2.6. Let $\kappa$ be regular and uncountable; Let $\bar{G}=\left\langle G_{\alpha}\right\rangle_{\alpha<\kappa}$ be a filtration of a free group $G$ (with universe $\kappa$ ). The bases of $G$ and the club subsets of $\operatorname{Div}(\bar{G})$ are equicomputable modulo $\bar{G}$. That is:

- If $C$ is a club subset of $\operatorname{Div}(\bar{G})$ then there is a basis $B$ of $G$ such that $B \leqslant \kappa{ }_{\kappa} C \oplus \bar{G}$

- If $B$ is a basis of $G$ then there is a club subset $C$ of $\operatorname{Div}(\bar{G})$ such that $C \leqslant \kappa B \oplus \bar{G}$.

The reductions in both directions are uniform. Briefly, given a club subset $C=$ $\left\{\alpha_{i}: i<\kappa\right\}$ of $\operatorname{Div}(\bar{G})$, we can present $G$ as the direct sum of $\kappa$-finite groups $H_{i}$ with $G_{\alpha_{i}}=\bigoplus_{j<i} H_{j}$, and combine bases of the groups $H_{i}$ to a basis of $G$. In the other direction, given a basis $B$ of $G$, we let $C$ be the collection of $\alpha$ such that $B \cap \alpha$ generates $G_{\alpha}$.

From now, we fix a regular uncountable cardinal $\kappa$. Recall the lexicographic ordering on $2^{\kappa}$ : $S<T$ if for the greatest $\delta<\kappa$ such that $S\lceil\delta=T\lceil\delta$ we have $\delta \in T \backslash S$. If $\left\langle S_{i}\right\rangle_{i \leqslant i^{*}}$ is a lexicographically nondecreasing sequence (for some ordinal $\left.i^{*}\right), i<j \leqslant i^{*}$ and $S_{i}\left\lceil\delta=S_{j}\left\lceil\delta\right.\right.$ for some $\delta<\kappa$, then $S_{r} \uparrow \delta$ is constant for all $r \in[i, j]$.

The lexicographic ordering is complete. A lexicographically nondecreasing sequence $\left\langle S_{i}\right\rangle_{i \leqslant i^{*}}$ is continuous if for all limit $j \leqslant i^{*}, S_{j}$ is the least upper bound of $\left\langle S_{i}\right\rangle_{i<j}$. This means that if $j \leqslant i^{*}$ is a limit, $\delta<\kappa$ and $S_{i}\lceil\delta$ is constant for some final segment of $i<j$, then $S_{i}\lceil\delta+1$ is also constant for some final segment of $i<j$.

A nondecreasing approximation of a set $S \subseteq \kappa$ is a uniformly $\kappa$-computable sequence $\left\langle S_{i}\right\rangle_{i<\kappa}$ which is lexicographically nondecreasing such that $S=\sup _{i<\kappa} S_{i}$. Sets which have nondecreasing approximations are called $\kappa$-left-c.e. If $S$ is $\kappa$-left-c.e. then it has a nondecreasing approximation which is also continuous. 
We say that a set $S \subseteq \kappa$ is nowhere stationary if for all $\alpha \leqslant \kappa$ of uncountable cofinality, $S \cap \alpha$ is nonstationary in $\alpha$.

To avoid repetition, we make the following definition.

Definition 2.7. We say that a nowhere stationary, $\kappa$-left-c.e. set $S \subseteq \kappa$ is nicely thin if there is a continuous nondecreasing approximation $\left\langle S_{i}\right\rangle_{i<\kappa}$ of $S$ such that for all $i<\kappa, S_{i}$ is nowhere stationary, and every $\alpha \in S_{i}$ is a limit ordinal of countable cofinality.

As promised, the following proposition distills the algebraic aspects of our construction.

Proposition 2.8. If $S \subset \kappa$ is nicely thin, then there is a $\kappa$-computable free abelian group $G$ and a $\Delta_{2}^{0}\left(L_{\kappa}\right)$ filtration $\bar{G}$ of $G$ such that $\operatorname{Div}(\bar{G})=\kappa \backslash S$.

The second part of the proof is:

Proposition 2.9. Let $X$ be $\Delta_{1}^{1}\left(L_{\kappa}\right)$. There is a nicely thin set $S \subseteq \kappa$ which intersects every $X$-computable club set.

Proof of Theorem 1.1, given Propositions 2.8 and 2.9. Let $X$ be $\Delta_{1}^{1}\left(L_{\kappa}\right)$; we may assume that $X \geqslant_{\kappa} \emptyset^{\prime}$. Let $S \subseteq \kappa$ be a nicely thin set given by Proposition 2.9. Let $G$ and $\bar{G}$ be given by Proposition 2.8 from this set $S$. Since $\bar{G}$ is $\Delta_{2}^{0}\left(L_{\kappa}\right)$ and $X \geqslant_{\kappa} \emptyset^{\prime}$, we have $X \geqslant_{\kappa} \bar{G}$. Suppose that $B$ is a basis of $G$. By Proposition 2.6, there is a club set $C \subseteq \operatorname{Div}(\bar{G})$ such that $C \leqslant{ }_{\kappa} B \oplus \bar{G}$. So $C$ is disjoint from $S=\kappa \backslash \operatorname{Div}(\bar{G})$, whence $C{ }_{\kappa} X$. Hence $B \Varangle_{\kappa} X$ as well.

The next two sections are devoted to the proofs of Propositions 2.8 and 2.9, respectively.

\section{BuILDing GROUPS FROM LEFT-C.E. SETS}

In this section we prove Proposition 2.8. The main tool we use is the "twisting" of an increasing $\omega$-sequence of free groups, which is Prop. 2.16 of [7].

Proposition 3.1. There is a $\kappa$-computable process which given a $\kappa$-finite increasing $\omega$-sequence $\left\langle H_{n}\right\rangle$ of free groups such that $H_{n} \mid H_{n+1}$ for all $n$, produces a $\kappa$-finite free group $G \supseteq H_{\omega}=\bigcup_{n} H_{n}$ (with $|G|=\left|H_{\omega}\right|$ ) such that every $H_{n}$ detaches in $G$ but $H_{\omega}$ does not detach in $G$.

This construction relies on a copy $G$ of $\mathbb{Z}^{(\omega+1)}$ such that $G / \mathbb{Z}^{(\omega)}$ is not free; Pontryagin's criterion implies that each finitely generated subgroup of $\mathbb{Z}^{(\omega)}$ detaches in $G$. In Proposition 3.1, note that each $H_{n}$ detaches in $H_{\omega}$.

First, we show how to prove Proposition 2.8 in the simpler case when $S$ is $\kappa$ computable (rather than merely $\kappa$-left-c.e.). We can then produce both $G$ and $\bar{G}$ to be $\kappa$-computable. The construction follows the proof of [7, Thm.3.1]. We define the sequence $\left\langle G_{\alpha}\right\rangle_{\alpha<\kappa}$ by effective $\kappa$-recursion $\left(\Sigma_{1}\right.$ recursion over $\left.L_{\kappa}\right)$. During the construction we ensure that each $G_{\alpha}$ is free, and for all $\beta<\alpha$, if $\beta \notin S$ then $G_{\beta} \mid G_{\alpha}$. The construction has three cases.

1. $\alpha$ is a limit: We must define $G_{\alpha}=\bigcup_{\beta<\alpha} G_{\alpha}$. Since $S \cap \alpha$ is nonstationary in $\alpha$ and $\operatorname{Div}\left(\left\langle G_{\beta}\right\rangle_{\beta<\alpha}\right) \supseteq \alpha \backslash S, G_{\alpha}$ is free (Proposition 2.4). Further, if $\beta \in \alpha \backslash S$ then as $\beta \in \operatorname{Div}\left(\left\langle G_{\beta}\right\rangle_{\beta<\alpha}\right)$, we have $G_{\beta} \mid G_{\alpha}$ (Remark 2.5). 
2. Defining $G_{\alpha+1}$ when $\alpha \notin S$ : We let $G_{\alpha+1}=G_{\alpha} \oplus \mathbb{Z}$. Since $G_{\alpha}$ is free, so is $G_{\alpha+1}$. We certainly have $G_{\alpha} \mid G_{\alpha+1}$, and for all $\beta \in \alpha \backslash S$, by induction, $G_{\beta} \mid G_{\alpha}$, and so $G_{\beta} \mid G_{\alpha+1}$ as well.

3. Defining $G_{\alpha+1}$ when $\alpha \in S$ : Since $\alpha \in S, \operatorname{cf}(\alpha)=\omega$, so we can effectively find a cofinal $\omega$-sequence $\left\langle\beta_{n}\right\rangle$ in $\alpha$; by replacing each $\beta_{n}$ by its successor, we may assume that each $\beta_{n} \notin S$. Thus, by induction, for all $n, G_{\beta_{n}} \mid G_{\beta_{n+1}}$, and $G_{\alpha}=\bigcup_{n} G_{\beta_{n}}$. We then apply Proposition 3.1 to the sequence $\left\langle G_{\beta_{n}}\right\rangle$ to obtain a free group $G_{\alpha+1}$ extending $G_{\alpha}$ such that $G_{\alpha} \nmid G_{\alpha+1}$ for all $n$. If $\beta \leqslant \alpha$ and $\beta \notin S$, then $\beta<\alpha$; for sufficiently large $n$ we have $\beta_{n}>\beta$. By induction, $G_{\beta} \mid G_{\beta_{n}}$, and by construction, $G_{\beta_{n}} \mid G_{\alpha+1}$, so $G_{\beta} \mid G_{\alpha+1}$ as required.

We define $G=G_{\kappa}=\bigcup_{\alpha<\kappa} G_{\alpha}$. It is $\kappa$-computable since it is the union of a $\kappa$-computable sequence of $\kappa$-finite groups. Case 1 above holds for $\kappa$ as well: $\operatorname{Div}\left(\left\langle G_{\alpha}\right\rangle\right) \supseteq \kappa \backslash S$; since $S$ is nonstationary in $\kappa, G$ is free. On the other hand, for all $\alpha \in S$, by construction, $G_{\alpha} \nmid G_{\alpha+1}$, so $\beta \notin \operatorname{Div}\left(\left\langle G_{\alpha}\right\rangle\right)$; overall, we see that $\operatorname{Div}\left(\left\langle G_{\alpha}\right\rangle\right)=\kappa \backslash S$ as required.

We now consider the general case, when $S$ is $\kappa$-left-c.e. The construction is an elaboration on the proof of [7, Thm.3.2]. The idea is that if at stage $s<\kappa$ we see a a change in $S$ at some small $\alpha<s$, then we keep the group that we have constructed so far, but "squash" the filtration so that the old $G_{s}$ becomes a subgroup of the new $G_{\alpha}$. The fact that the smallest such change is into $S$ allows us to introduce a twist (otherwise we would need to remove a twist, which is impossible).

Let $S \subset \kappa$ be nicely thin, and let $\left\langle S_{t}\right\rangle_{t<\kappa}$ be an approximation satisfying Definition 2.7. For brevity, we let $S_{\kappa}=S$.

We now construct, by recursion on $t \leqslant \kappa$, filtrations $\overline{G^{t}}=\left\langle G_{\alpha}^{t}\right\rangle_{\alpha \leqslant \gamma(t)}$ (for ordinals $\gamma(t) \leqslant t)$, satisfying:

(a) $G_{\gamma(s)}^{s} \subseteq G_{\gamma(t)}^{t}$ for $s \leqslant t \leqslant \kappa$, and if $t$ is a limit then $G_{\gamma(t)}^{t}=\bigcup_{s<t} G_{\gamma(s)}^{s}$. That is, the sequence $\left\langle G_{\gamma(s)}^{s}\right\rangle_{s \leqslant \kappa}$ is increasing and continuous.

(b) $G_{\gamma(t)}^{t}$ is free and $\operatorname{Div}\left(\overline{G^{t}}\right)=\gamma(t) \backslash S_{t}$ for all $t \leqslant \kappa$. Indeed, for all $\beta<\gamma(t)$, if $\beta \notin S_{t}$ then $G_{\beta}^{t} \mid G_{\gamma(t)}^{t}$, while if $\beta \in S_{t}$ then $G_{\beta}^{t} \nmid G_{\beta+1}^{t}$.

(c) For all $s \leqslant t \leqslant \kappa$ and $\alpha \leqslant \gamma(s)$, if $S_{s}\left\lceil\alpha=S_{t} \uparrow \alpha\right.$ then $\alpha \leqslant \gamma(t)$ and $G_{\alpha}^{s}=G_{\alpha}^{t}$.

(d) For all $s<t \leqslant \kappa$ and $\alpha \leqslant \gamma(t)$, if $S_{s}\left\lceil\alpha \neq S_{t}\left\lceil\alpha\right.\right.$ then $G_{\gamma(s)}^{s} \subseteq G_{\alpha}^{t}$.

We first consider the successor case. Suppose that $\overline{G^{s}}$ has been defined for all $s \leqslant t$; we show how to define $\overline{G^{t+1}}$. First, we let

$$
\delta=\max \left\{\beta \leqslant \gamma(t): S_{t}\left\lceil\beta=S_{t+1}\lceil\beta\} .\right.\right.
$$

For $\beta \leqslant \delta$ we let $G_{\beta}^{t+1}=G_{\beta}^{t}$. We let $\gamma(t+1)=\delta+1$, and so we need to define $G_{\delta+1}^{t+1}$. There are two sub-cases. If $\delta \notin S_{t+1}$, then $\delta \notin S_{t}$, and so the maximality of $\delta$ shows that $\delta=\gamma(t)$. In this sub-case we simply let $G_{\delta+1}^{t+1}=G_{\delta}^{t} \oplus \mathbb{Z}$.

Suppose that $\delta \in S_{t+1}$. Either $\delta=\gamma(t)$, or $\delta \notin S_{t}$ (by the maximality of $\delta$ ). In either case, $G_{\delta}^{t} \mid G_{\gamma(t)}^{t}$. Write $G_{\gamma(t)}^{t}=G_{\delta}^{t} \oplus K$ (where $K$ may be trivial). Since $\operatorname{cf}(\delta)=\omega\left(\right.$ as $\left.\delta \in S_{t+1}\right)$, we choose a sequence $\left\langle\beta_{n}\right\rangle$ cofinal in $\delta$ and disjoint from $S_{t+1}$. By (b), $G_{\beta_{n}}^{t} \mid G_{\beta_{n+1}}^{t}$ for all $n<\omega$, so we appeal to Proposition 3.1 to get a free group $G \supset G_{\delta}^{t}$ in which every $G_{\beta_{n}}$ detaches, but $G_{\delta}^{t} \nmid G$. We let $G_{\delta+1}^{t+1}=G \oplus K$. 
By design, $G_{\gamma(t)}^{t} \subseteq G_{\delta+1}^{t+1}=G_{\gamma(t+1)}^{t+1}$, verifying (a) for stage $t+1$. We verify (b). For $\beta<\delta$, if $\beta \in S_{t+1}$ then $\beta \in S_{t}$ and then $G_{\beta}^{t} \nmid G_{\beta+1}^{t}$, which shows that $\beta \notin \operatorname{Div}\left(\overline{G^{t+1}}\right)$; if $\beta \notin S_{t+1}$ then $\beta \notin S_{t}$. If $\delta \notin S_{t+1}$ then $G_{\beta}^{t}\left|G_{\delta}^{t}\right| G_{\delta+1}^{t+1}$, showing that $\beta \in \operatorname{Div}\left(\overline{G^{t+1}}\right)$. If $\delta \in S_{t+1}$ then for some $n, \beta<\beta_{n}$, and $G_{\beta}^{t}\left|G_{\beta_{n}}^{t}\right| G_{\delta+1}^{t+1}$, showing that $\beta \in \operatorname{Div}\left(\overline{G^{t+1}}\right)$. (c) between $t$ and $t+1$ follows from the definition of $\delta$. In general, for $s<t$, if $\alpha \leqslant \gamma(s)$ and $S_{s}\left\lceil\alpha=S_{t+1}\left\lceil\alpha\right.\right.$, then $S_{s}\left\lceil\alpha=S_{t}\lceil\alpha\right.$ as well; by induction, $\alpha \leqslant \gamma(t)$, and so $\alpha \leqslant \delta$ and $G_{\alpha}^{s}=G_{\alpha}^{t}=G_{\alpha}^{t+1}$. For (d), let $s<t+1, \alpha \leqslant \gamma(t+1)$, and suppose that $S_{s}\left\lceil\alpha \neq S_{t+1}\lceil\alpha\right.$; we need to show that $G_{\gamma(s)}^{s} \subseteq G_{\alpha}^{t+1}$. In case $\alpha=\gamma(t+1)$, this follows from (a). Otherwise, $\alpha \leqslant \delta$, and so $\alpha \leqslant \gamma(t)$, and $G_{\alpha}^{t+1}=G_{\alpha}^{t}$. By the definition of $\delta$, it must be that $S_{s}\left\lceil\alpha \neq S_{t}\lceil\alpha\right.$. Then by induction, $G_{\gamma(s)}^{s} \subseteq G_{\alpha}^{t}$.

Now suppose that $t \leqslant \kappa$ is a limit ordinal, and suppose that the filtrations $\overline{G^{s}}$ have been defined for all $s<t$, and satisfy the conditions described. We now describe how to define $\overline{G^{t}}$. Let

$$
\Delta=\left\{\beta: \exists s<t\left[\beta \leqslant \gamma(s) \&\left(S_{s}\left\lceil\beta=S_{t} \uparrow \beta\right)\right]\right\} .\right.
$$

If $s<t$ witnesses that $\beta \in \Delta$, then $s$ witnesses that every $\epsilon<\beta$ is in $\Delta$ as well, and so $\Delta$ is actually an ordinal (a set of ordinals closed downwards). We let $\gamma(t)=\Delta$.

We argue that $\Delta$ is in fact a limit ordinal. This relies on the approximation $\left\langle S_{s}\right\rangle$ being continuous. Suppose, for a contradiction, that $\delta=\max \Delta=\Delta-1$ exists. Let $s<t$ witness that $\delta \in \Delta$. Then $S_{r}\lceil\delta$ is constant for $r \in[s, t]$. By definition of $\Delta$ we have $\delta \leqslant \gamma(s)$. Since (c) holds by induction, for all $r \in[s, t)$ we have $\delta \leqslant \gamma(r)$, that is, every $r \geqslant s$ also witnesses that $\delta \in \Delta$. Since the approximation is continuous, for sufficiently late $r$, we have $S_{r}(\delta)=S_{t}(\delta)$, that is, $S_{r}\lceil\delta+1$ is constant on a final segment of $r<t$. So we may assume that $S_{s}\left\lceil\delta+1=S_{t}\lceil\delta+1\right.$. Now by our instructions for the successor case, we have $\gamma(s+1) \geqslant \delta+1$, so stage $s+1$ witnesses that $\delta+1 \in \Delta$, a contradiction.

Now for all $\beta<\gamma(t)=\Delta$, we define $G_{\beta}^{t}=G_{\beta}^{s}$ for any $s<t$ witnessing that $\beta \in \Delta$; again, as $S_{r}\left\lceil\beta=S_{t} \uparrow \beta\right.$ for all $r \in[s, t)$, by (c) $G_{\beta}^{r}$ is constant for all such $r$, so $G_{\beta}^{t}$ is well-defined, and $\left\langle G_{\beta}^{t}\right\rangle_{\beta<\gamma(t)}$ is increasing and continuous; we let $G_{\gamma(t)}^{t}=\bigcup_{\beta<\gamma(t)} G_{\beta}^{t}$, and this defines $\overline{G^{t}}$, which is indeed a filtration. We verify that the conditions above hold for $t$.

(c) is by construction. For (d), let $s<t$ and $\alpha \leqslant \gamma(t)$, and suppose that $S_{s}\left\lceil\alpha \neq S_{t}\lceil\alpha\right.$. As above, we now assume that $\alpha<\gamma(t)$, as the case $\alpha=\gamma(t)$ will follow from (a), which we will soon verify. Since $\alpha \in \Delta$, find some $r<t$ such that $S_{r}\left\lceil\alpha=S_{t}\left\lceil\alpha\right.\right.$ and $\alpha \leqslant \gamma(r)$. Then $s<r<t$. By induction, $G_{\gamma(s)}^{s} \subseteq G_{\alpha}^{r}$, and $G_{\alpha}^{r}=G_{\alpha}^{t}$.

For (a), Let $s<t$. Since $\gamma(t) \notin \Delta$, and $\gamma(t)$ is a limit, there is some $\beta<\gamma(t)$ such that $S_{s} \uparrow \beta \neq S_{t} \uparrow \beta$. Again let $r \in(s, t)$ such that $S_{r} \uparrow \beta=S_{t} \uparrow \beta$ and $\beta \leqslant \gamma(r)$. By induction,

as required.

$$
G_{\gamma(s)}^{s} \subseteq G_{\beta}^{r}=G_{\beta}^{t} \subseteq G_{\gamma(t)}^{t}
$$

Finally, we verify (b). Let $\beta<\gamma(t)$. Suppose that $\beta \in S_{t}$. Take $s<t$ witnessing that $\beta+1 \in \Delta$. Then $\beta \in S_{s}$ and by induction, $G_{\beta}^{s} \nmid G_{\beta+1}^{s}$; and $G_{\beta}^{t}=G_{\beta}^{s}$ and $G_{\beta+1}^{t}=G_{\beta+1}^{s}$. Suppose that $\beta \notin S_{t}$, and let $\delta \in(\beta, \gamma(t))$. Let $s<t$ witness that $\delta \in \Delta$. Then $\delta \notin S_{s}$, and so $G_{\beta}^{s} \mid G_{\delta}^{s}$; so $G_{\beta}^{t} \mid G_{\delta}^{t}$, showing that $\beta \in \operatorname{Div}\left(\left\langle G_{\alpha}^{t}\right\rangle_{\alpha<\gamma(t)}\right)$. 
Now by assumption, the set $S_{t}$ in nonstationary in $\gamma(t)$, and so $G_{\gamma(t)}^{t}$ is free, and $\operatorname{Div}\left(\overline{G^{t}}\right)=\gamma(t) \backslash S_{t}$.

This completes the construction. There is one thing left to show: that $\gamma(\kappa)=$ $\kappa$. This fundamentally follows from the regularity of $\kappa$, which implies that the approximation $\left\langle S_{t}\right\rangle$ of $S=S_{\kappa}$ is tame: for all $\beta<\kappa$ there is some $t<\kappa$ such that $S_{t} \uparrow \beta=S_{\kappa} \uparrow \beta$. [This is proved by induction on $\beta$; if this is known for all $\alpha<\beta$, then the function taking such $\alpha$ to the least $t$ for which $S_{t}\left\lceil\alpha=S_{\kappa}\lceil\alpha\right.$ must be bounded below $\kappa$.] So now we show by induction on $\beta<\kappa$ that $\beta \leqslant \gamma(s)$ for some $s$ for which $S_{s} \uparrow \beta=S_{\kappa} \uparrow \beta$ (which by (c) for $s$ and $\kappa$ implies that $\beta \leqslant \gamma(\kappa)$ ). If this is known for $\beta$, then by taking a sufficiently late $s$, we may assume that $S_{s} \uparrow \beta+1=S_{\kappa} \uparrow \beta+1$ as well; then by construction, $\beta+1 \leqslant \gamma(s+1)$. Now suppose that $\beta$ is a limit ordinal. For all $\alpha<\beta$, let $s_{\alpha}$ be least $s$ such that $\alpha \leqslant \gamma(s)$ and $S_{s}\left\lceil\alpha=S_{\kappa}\left\lceil\alpha\right.\right.$. Then the sequence $\left\langle s_{\alpha}\right\rangle_{\alpha<\beta}$ is nondecreasing; let $s^{*}=\sup _{\alpha<\beta} s_{\alpha}$. Then $S_{s^{*}} \uparrow \beta=S_{\kappa} \uparrow \beta$, and by (c), for all $\alpha<\beta, \alpha \leqslant \gamma\left(s^{*}\right)$, so $\beta \leqslant \gamma\left(s^{*}\right)$.

The restriction of the construction to $t<\kappa$ is $\kappa$-computable. In particular, the sequence $\left\langle G_{\gamma(s)}^{s}\right\rangle$ is $\kappa$-computable, and so its union, which is $G_{\kappa}^{\kappa}$, is $\kappa$-computable; by (b) at $t=\kappa$, it is free. The filtration $\left\langle G_{\alpha}^{\kappa}\right\rangle_{\alpha<\kappa}$ is $\Delta_{2}^{0}\left(L_{\kappa}\right)$ and by (b), $\operatorname{Div}\left(\overline{G^{\kappa}}\right)=$ $\kappa \backslash S_{\kappa}$ as required. This completes the proof of Proposition 2.8. ${ }^{1}$

\section{Constructing fat thin SETs}

In this section we prove Proposition 2.9. As in the previous section, we first consider the construction under some simplifying assumptions. First, we review the basic tools of fine-structure theory that were used in [7] and will use again below. They are taken from Jensen's original paper [8].

Definition 4.1. The class $E$ consists of all the singular ordinals $\alpha$ such that for some $\beta>\alpha$ :

- $L_{\beta}=\mathrm{ZF}^{-}$;

- $\alpha$ is the greatest cardinal of $L_{\beta}$;

- there exists $p \in L_{\beta}$ such that $L_{\beta}$ is the least fully elementary substructure $M \prec L_{\beta}$ with $p \in M$ and $M \cap \alpha$ transitive.

Each $\alpha \in E$ has countable cofinality; in fact, if $\alpha \in E$, witnessed by $\beta$, then there is a cofinal $\omega$-sequence in $\alpha$ definable over $L_{\beta+1}$. Thus, once we see that an ordinal $\alpha$ is singular, we can effectively tell if $\alpha \in E$ or not. Thus, for any regular $\kappa$, $E \cap \kappa$ is $\kappa$-c.e.; if $\kappa$ is a successor cardinal, then $E \cap \kappa$ is $\kappa$-computable.

The following lemma is used to produce elements of $E$ :

Lemma 4.2. Let $\kappa$ be regular and uncountable; let $q \in L_{\kappa^{+}}$. Let $M$ be the least elementary substructure of $L_{\kappa^{+}}$such that $q \in M$ and $M \cap \kappa$ is transitive. Let $\pi: M \rightarrow L_{\beta}$ be the Mostowski collapse; let $\alpha=\pi(\kappa)=M \cap \kappa$. Then $\alpha \in E$, witnessed by $\beta$.

The deep fact about $E$ that is used throughout is [8, Thm.5.1]:

\footnotetext{
${ }^{1}$ Note that there is no reason to believe that $\operatorname{Div}\left(\left\langle G_{\gamma(s)}^{s}\right\rangle\right)=\kappa \backslash S$; they agree on a club, but that club may fail to be $\kappa$-computable, it is merely $\Delta_{2}^{0}\left(L_{\kappa}\right)$.
} 
Theorem 4.3 (Jensen). The class $E$ does not reflect at any singular ordinal. That is, if $\alpha$ is singular then $E \cap \alpha$ is nonstationary in $\alpha$.

On the other hand, $E$ is stationary in every regular cardinal.

Toward the full proof of Proposition 2.9, we give the proof when $\kappa$ is asuccessor cardinal. In that case, $E \cap \kappa$ is $\kappa$-computable, and so we can make the desired set $S$ $\kappa$-computable as well. To simplyfy even further, rather than meeting $X$-computable clubs for some possibly quite complicated $X$, we consider a simpler collection of sets, namely the first-order definable ones. We thus prove:

Proposition 4.4. Let $\kappa$ be a successor cardinal. There is a $\kappa$-computable set $S$ which is nowhere stationary but intersects every club of $\kappa$ which is first-order definable over $L_{\kappa}$.

This implies that there is a $\kappa$-computable free group with no first-order definable basis.

Proof. The set $S$ is constructed by recursion. At stage $\delta<\kappa$, we will have already defined $S\lceil\delta$. If $\delta \notin E$ then $\delta \notin S$. Suppose that $\delta \in E$. Then we let $\delta \in S$ if and only if there is some $C \subseteq \delta$, closed and unbounded in $\delta$, which is first-order definable over $L_{\delta}$, which is disjoint from $S \cap \delta$.

Because $E$ is $\kappa$-computable, the construction is $\kappa$-computable, and so $S$ is $\kappa$ computable.

Let us show that $S$ intersects every club of $\kappa$, first-order definable over $L_{\kappa}$. Let $C$ be such a club. Let $M$ be the smallest elementary substructure of $L_{\kappa^{+}}$ which contains the parameters used for the definition of $C$, and such that $M \cap \kappa$ is transitive. Let $\pi: M \rightarrow L_{\beta}$ be the Mostowski collapse, and let $\alpha=\pi(\kappa)=M \cap \kappa$. By Lemma 4.2, $\alpha \in E$. Now $\pi(C)=C \cap \alpha$ is a club of $\alpha$ which is first-order definable over $L_{\alpha}=\pi\left(L_{\kappa}\right)$. Also note that $\alpha \in C$ (as $C$ is closed). If $\pi(C) \cap(S \cap \alpha) \neq \emptyset$ then some $\gamma<\alpha$ is an element of $S \cap C$, in which case we are done. Otherwise, by construction, we put $\alpha$ into $S$, so in this case $\alpha \in C \cap S$.

It remains to show that $S$ is nowhere stationary. By construction, $S \subseteq E$, and $E$ does not reflect at any singular ordinal (Theorem 4.3), so it suffices to show that $S \cap \lambda$ is nonstationary in $\lambda$, for every regular cardinal $\lambda \leqslant \kappa$.

By constructing an increasing, continuous sequence of elementary submodels of $L_{\kappa^{+}}$, we obtain a closed set $D \subseteq \kappa$ such that $D \cap \lambda$ is unbounded in $\lambda$ for every regular $\lambda \leqslant \kappa$, and such that for all $\alpha \in D$ there is a model $M_{\alpha} \prec L_{\kappa^{+}}$with $\alpha=M \cap \kappa$. It suffices to show that $D \cap S=\emptyset$. Let $\alpha \in D$; let $\pi: M_{\alpha} \rightarrow L_{\beta}$ be the Mostowski collapse. Let $C \subseteq \alpha$ be a club of $\alpha$, first-order definable over $L_{\alpha}$. Then $C \in L_{\beta}$, and $\pi^{-1}(C)$ is a club of $\kappa$, first-order definable over $L_{\kappa}$. We have just proved that $S \cap \pi^{-1}(C)=\emptyset$. Note that $S \in M_{\alpha}$ (as it is definable over $L_{\kappa}$ ). By elementarity of $M_{\alpha}$, there is some $\gamma<\alpha$ in $\pi^{-1}(C) \cap S$. Then $\gamma \in C \cap(S \cap \alpha)$. Thus, $S \cap \alpha$ meets all clubs of $\alpha$ which are first-order definable over $L_{\alpha}$, and so even if $\alpha \in E$, the construction would instruct us to keep $\alpha$ out of $S$.

Now for the full proof of Proposition 2.9, we need to overcome two obstacles:

- If $\kappa$ is not a successor cardinal, we need to deal with the fact that $E \cap \kappa$ is not $\kappa$-computable, but merely $\kappa$-c.e.

- We need to diagonalise against all $X$-computable clubs for some $X$ which is $\Delta_{1}^{1}\left(L_{\kappa}\right)$, not just first-order definable ones. 
For the first difficulty, we fix a $\kappa$-computable enumeration $\left\langle E_{t}\right\rangle_{t<\kappa}$ of $E$, and repeat the construction above at each stage $t \leqslant \kappa$, giving us a set $S_{t}$ for each such $t$; we will show that this is an approximation as required. For the second, we use a technique of approximating (or reflecting) $\Delta_{1}^{1}$ sets that was used by Johnston [9, Thm.4.43] and S. Friedman and his co-authors [4, Lem.2.5].

Fix a $\Delta_{1}^{1}\left(L_{\kappa}\right)$ set $X$. Thus, there are two first-order formulas $\varphi$ and $\psi$, with parameters in $L_{\kappa}$, such that $(\exists Y) \psi(-, Y)$ and $(\exists Y) \varphi(-, Y)$ define $X$ and its complement respectively, where the variable $Y$ ranges over subsets of $\kappa$, and for each $Y \subseteq \kappa$, the formula $\varphi(-, Y)$ is evaluated in the structure $\left(L_{\kappa} ; \in, Y\right)$ (with $Y$ interpreting a unary predicate).

Fix ordinals $\alpha<\beta$ such that $L_{\beta}=\mathrm{ZF}^{-}$and $\alpha$ is regular in $L_{\beta}$ and is the largest cardinal of $L_{\beta}$. Also assume that the parameters used for the formulas $\varphi$ and $\psi$ are elements of $L_{\alpha}$. We let

$$
A_{\alpha}^{\beta}=\left\{\gamma<\alpha:\left(\exists y \in L_{\beta}\right) y \subseteq \alpha \& L_{\alpha}=\varphi(\gamma, y)\right\}
$$

and

$$
B_{\alpha}^{\beta}=\left\{\gamma<\alpha:\left(\exists y \in L_{\beta}\right) y \subseteq \alpha \& L_{\alpha}=\psi(\gamma, y)\right\} .
$$

If $\alpha \in E$ the we write $A_{\alpha}$ and $B_{\alpha}$ for $A_{\alpha}^{\beta}$ and $B_{\alpha}^{\beta}$ for the unique $\beta$ which witnesses that $\alpha \in E$. We say that $\alpha \in E$ is good if $B_{\alpha}=\alpha \backslash A_{\alpha}$. In this case, $A_{\alpha}$ is our guess for $X$ at level $\alpha$. The guess is correct on a club. This follows from:

Lemma 4.5. Suppose that $M$ is an elementary substructure of $L_{\kappa^{+}}$(containing the parameters for $\varphi$ and $\psi$ ), and $M \cap \kappa \in \kappa$. Let $\alpha=M \cap \kappa$, and let $L_{\beta}$ be the Mostowski collapse of $M$. Then $B_{\alpha}^{\beta}=\alpha \backslash A_{\alpha}^{\beta}$, and $A_{\alpha}^{\beta}=X \cap \alpha$.

We now provide the proof of Proposition 2.9. Let $\kappa$ be regular; fix $X, \varphi$ and $\psi$ as above. Let $\left\langle E_{t}\right\rangle$ be a $\kappa$-computable enumeration of $E \cap \kappa$ : for all $t \leqslant \kappa, E_{t}$ is the collection of $\alpha<t$ which are witnessed to be in $E$ by some $\beta<t$. The facts about this enumeration that we use are:

(1) If $s \leqslant t \leqslant \kappa$ then $E_{s} \subseteq E_{t}$; if $t \leqslant \kappa$ is limit then $E_{t}=\bigcup_{s<t} E_{s} ; E_{\kappa}=E \cap \kappa$.

(2) For any cardinal $\lambda<\kappa$, for all $t \geqslant \lambda, E_{t} \cap \lambda=E \cap \lambda$.

Now for each $t \leqslant \kappa$ we define a set $S_{t} \subseteq E_{t}$ by recursion. For $\alpha<t$, if $S_{t}\lceil\alpha$ has already been defined, then we set $\alpha \in S_{t}$ if and only if $\alpha \in E_{t}, \alpha$ is good, and there is a club of $\alpha$, disjoint from $S_{t} \cap \alpha$, which is $\Delta_{1}^{0}$ definable in the structure $\left(L_{\alpha} ; \in, A_{\alpha}\right)$.

We first observe that $S_{\kappa}$ intersects every $X$-computable club. Let $C$ be an $X$ computable club. As above, let $M$ be a minimal elementary submodel of $L_{\kappa^{+}}$ containing both the parameters for the definitions of $X$ and its complement, and for the reduction of $C$ to $X$, such that $M \cap \kappa \in \kappa$; let $\alpha=M \cap \kappa$. Let $\pi: M \rightarrow L_{\beta}$ be the Mostowski collapse. By Lemma 4.2, $\alpha \in E$, witnessed by $\beta$. The reduction of $C$ to $X$, and the fact that $A_{\alpha}=X \cap \alpha$ (Lemma 4.5) shows that $C \cap \alpha$ is $\Delta_{1}^{0}$-definable in $\left(L_{\alpha} ; \in, A_{\alpha}\right)$. Thus either $C \cap S_{\kappa} \cap \alpha$ is nonempty, or the construction puts $\alpha$ into $S_{\kappa}$, and so $\alpha \in S_{\kappa} \cap C$.

Next, we show that $S_{\kappa}$ is nowhere stationary. Since $S_{\kappa} \subseteq E$, it again suffices to consider rgular cardinals $\lambda \leqslant \kappa$, and we use the same club $D$ as above: $D \cap \lambda$ is a club of $\lambda$ for all regular $\lambda \leqslant \kappa$, and for all $\alpha \in D$ there is some $M_{\alpha} \prec L_{\kappa^{+}}$with $\alpha=M_{\alpha} \cap \kappa$. We show that $D \cap S_{\kappa}=\emptyset$. Let $\alpha \in D$. To verify that $\alpha \notin S$, we may assume that $\alpha \in E$ and is good. Let $\beta$ witness that $\alpha \in E$, and let $\pi: M_{\alpha} \rightarrow L_{\gamma}$ 
be the Mostowski collapse. Since $L_{\gamma}$ thinks that $\alpha$ is regular (even with respect to sequences definable over $L_{\gamma}$ ), we have $\gamma \leqslant \beta$. By Lemma $4.5, B_{\alpha}^{\gamma}=\alpha \backslash A_{\alpha}^{\gamma}$ (and $A_{\alpha}^{\gamma}=X \cap \alpha$ ). On the other hand, because both $A_{\alpha}$ and $B_{\alpha}$ are defined by existential formulas, they are upwards absolute, which implies that $A_{\alpha}^{\gamma} \subseteq A_{\alpha}^{\beta}=A_{\alpha}$ and $B_{\alpha}^{\gamma} \subseteq B_{\alpha}^{\beta}=B_{\alpha}$. Since $\alpha$ is good, we must have $A_{\alpha}=A_{\alpha}^{\gamma}=X \cap \alpha$.

Let $C$ be a club of $\alpha$ which is $\Delta_{1}^{0}$-definable over $\left(L_{\alpha} ; \in, A_{\alpha}\right)$. Using the same definition over $L_{\kappa}$ with $X$ replacing $A_{\alpha}$ results with a club $\tilde{C}$ of $\kappa$ which is $X$ computable and such that $\pi(\tilde{C})=\tilde{C} \cap \alpha=C$. Now $S_{\kappa} \in M_{\alpha}$ and $\pi\left(S_{\kappa}\right)=S_{\kappa} \cap \alpha$; as $S_{\kappa} \cap \tilde{C} \neq \emptyset$ and $M_{\alpha} \prec L_{\kappa^{+}}$, as above we have $C \cap\left(S_{\kappa} \cap \alpha\right) \neq \emptyset$. So our instructions ensure that $\alpha \notin S$.

It follows that for all $t<\kappa, S_{t}$ is nowhere statonary as well. Since $S_{t} \subseteq E_{t} \subseteq E$, it again suffices to check that $S_{t} \cap \lambda$ is nonstationary in $\lambda$, when $\lambda \leqslant \kappa$ is regular. No if $\lambda>t$ then $S_{t} \subseteq t$ is bounded in $\lambda$, and so nonstationary. If $\lambda \leqslant t$ then as $E_{t} \cap \lambda=E \cap \lambda$, we can see that $S_{t} \cap \lambda=S_{\kappa} \cap \lambda$ (by induction on $\alpha<\lambda$ we see that $\left.S_{t} \cap \alpha=S_{\kappa} \cap \alpha\right)$. And we have already observed that $S_{\kappa} \cap \lambda$ is nonstationary in $\lambda$.

It remains to show that $\left\langle S_{t}\right\rangle_{t \leqslant \kappa}$ is lexicographically nondecreasing and is continuous. Let $s<t \leqslant \kappa$. Let $\delta<\kappa$ be such that $S_{s} \uparrow \delta=S_{t} \uparrow \delta$. If $\delta \in S_{s}$ then $\delta \in E_{s}$ and so $\delta \in E_{t}$; the same calculation that put $\delta$ into $S_{s}$ holds for $S_{t}$, so $\delta \in S_{t}$. Hence $S_{s} \leqslant S_{t}$ lexicographically.

Let $t \leqslant \kappa$ be a limit ordinal. Let $\delta<\kappa$ and $s<t$ such that $S_{s} \uparrow \delta=S_{t} \uparrow \delta$. Suppose that $\delta \in S_{t}$. So $\delta \in E_{t}$. Since $E_{t}=\bigcup_{r<t} E_{r}$, for sufficiently late $r$, we have $\delta \in E_{r}$; if $\delta \in E_{r}$ and $S_{r} \uparrow \delta=S_{t} \uparrow \delta$ then the same calculation that put $\delta$ into $S_{t}$ also puts $\delta$ into $S_{r}$. This shows that $S_{t}=\sup _{r<t} S_{r}$, and completes the proof of Proposition 2.9, and so of Theorem 1.1.

\section{FURTHER WORK}

Theorem 1.1 is optimal: R. Johnston has noticed (see [9]) that every $\kappa$-computable free group has a basis which is $\Delta_{1}^{1}\left(L_{\kappa}\right)$; namely, the $<_{L}$-least basis is $\Delta_{1}^{1}\left(L_{\kappa}\right)$.

However, one can study the general question of the "degree spectra" of bases of $\kappa$-computable free groups: what collections of $\kappa$-degrees can be realised as the collection of degrees computing bases for some $\kappa$-computable free group? In [7], the authors show that one cannot code much into bases; depending on $\kappa$, the most that can be coded into all bases of some group is either $\emptyset^{\prime}$ or $\emptyset^{\prime \prime}$. One can ask, though, for example, in view of Proposition 4.4, whether there is a $\kappa$-computable free group $G$ such that a $\kappa$-degree computes a basis for $G$ if and only if it is not first-order definable over $L_{\kappa}$ (not computable from $\emptyset^{(n)}$ for any $n$ ), and similarly, whether the non-arithmetic degrees (those not below $\emptyset^{(\alpha)}$ for any $\alpha<\kappa$ ) can be similarly realised.

\section{REFERENCES}

[1] Rodney Downey and Alexander G. Melnikov. Effectively categorical abelian groups. J. Algebra, 373:223-248, 2013.

[2] Paul C. Eklof. Methods of logic in abelian group theory. pages 251-269. Lecture Notes in Math., Vol. 616, 1977.

[3] Paul C. Eklof and Alan H. Mekler. Almost free modules, volume 65 of North-Holland Mathematical Library. North-Holland Publishing Co., Amsterdam, revised edition, 2002. Settheoretic methods.

[4] Ekaterina Fokina, Sy-David Friedman, Julia Knight, and Russell Miller. Classes of structures with universe a subset of $\omega_{1}$. J. Logic Comput., 23(6):1249-1265, 2013. 
[5] László Fuchs. Infinite abelian groups. Vol. I. Pure and Applied Mathematics, Vol. 36. Academic Press, New York-London, 1970.

[6] Noam Greenberg and Julia F. Knight. Computable structure theory on $\omega_{1}$ using admissibility. In Effective mathematics of the uncountable, volume 41 of Lect. Notes Log., pages 50-80. Assoc. Symbol. Logic, La Jolla, CA, 2013.

[7] Noam Greenberg, Dan Turetsky, and Linda Brown Westrick. Finding bases of uncountable free abelian groups is usually difficult. Trans. Amer. Math. Soc., 370(6):4483-4508, 2018.

[8] Ronald Björn Jensen. The fine structure of the constructible hierarchy. Ann. Math. Logic, 4:229-308; erratum, ibid. 4 (1972), 443, 1972. With a section by Jack Silver.

[9] Reese Johnston. Computability in uncountable binary trees. J. Symb. Log., 84(3):1049-1098, 2019.

[10] L. Pontrjagin. The theory of topological commutative groups. Ann. of Math. (2), 35(2):361$388,1934$.

School of Mathematics and Statistics, Victoria University of Wellington, WellingTON, New ZeAland

E-mail address: greenberg@msor.vuw.ac.nz

$U R L:$ http://homepages.mcs.vuw.ac.nz/ greenberg/

School of Mathematics and Statistics, Victoria University of Wellington, WellingTON, New Zealand

E-mail address: linus.richter@sms.vuw.ac.nz

The Hebrew University of Jerusalem, Einstein Institute of Mathematics, Edmond J. Safra Campus, Givat Ram, Jerusalem 91904, Israel

Department of Mathematics, Hill Center-Busch Campus, Rutgers, The State University of New Jersey, 110 Frelinghuysen Road, Piscataway, NJ 08854-8019 USA

E-mail address: shelah@math.huji.ac.il

School of Mathematics and Statistics, Victoria University of Wellington, WellingTON, New Zealand

E-mail address: dan.turetsky@msor.vuw.ac.nz

$U R L:$ https://homepages.ecs.vuw.ac.nz/ dan/ 